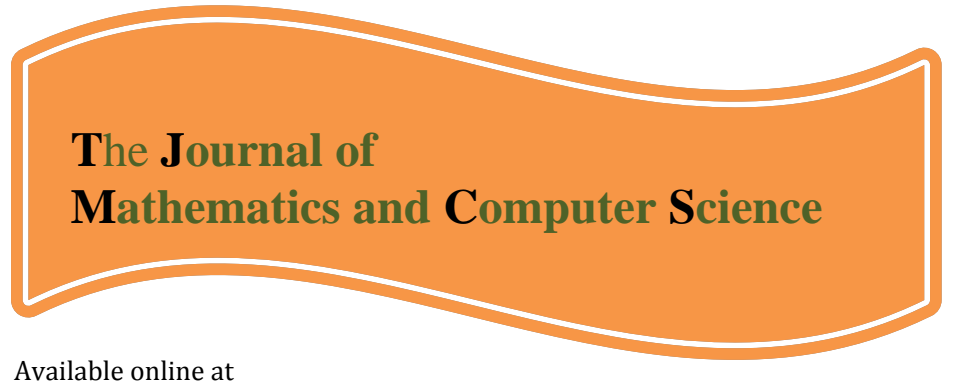

http://www.TJMCS.com

The Journal of Mathematics and Computer Science Vol .2 No.1 (2011) 88-99

\title{
A New Method for Modeling System Dynamics by Fuzzy Logic: Modeling of Research and Development in the National System of Innovation
}

\author{
Hassan Youssefi ${ }^{1, *}$, Vahid Saeid Nahaei ${ }^{2}$, Javad Nematian ${ }^{3}$ \\ Department of Industrial Engineering, University of Tabriz, P.O. Box 51666-14766, Tabriz, Iran, \\ Hassan.Youssefi66@Gmail.com \\ Department of Industrial Engineering, University of Tabriz, P.O. Box 51666-14766, Tabriz, Iran, \\ Nahaei@Iust.ac.ir \\ Department of Industrial Engineering, University of Tabriz, P.0. Box 51666-14766, Tabriz, Iran, \\ Jnematian@Tabrizu.ac.ir
}

Received: September 2010, Revised: December 2010

Online Publication: January 2011

\begin{abstract}
System Dynamics (SD) is an effective method for studying dynamic conditions and changes in complex systems. It has been used in domain of social, economic and human activities which deal with vague and inaccurate variables. In this paper, a new dynamic model of real world systems is designed based on the concept of system dynamic approach. Then relations among the variables in the model are defined as fuzzy if-then rules by using fuzzy logic method. For analyzing the model accurately and avoiding the extent of ambiguities, Fuzzy Inference System (FIS) will be designed. For this purpose, cycle of creation and absorption of knowledge in a National Innovation System has been analyzed via SD methodology and FIS results.
\end{abstract}

Keywords: System Dynamics, Fuzzy Logic, Fuzzy Inference System, National Innovation Systems, If-Then rules

\section{Introduction}

\footnotetext{
1,* Corresponding author : Bachelor of Science, Industrial Engineering

2 Assistant Professor of Industrial Engineering at University of Tabriz

3 Assistant Professor of Industrial Engineering at University of Tabriz
} 
System Dynamics (SD) is a significant approach that helps top management in the important problems and which is applied to issues ranging from corporate strategy to the dynamics of diabetes, from the cold war between the US and USSR to the combat between HIV and human immune system. Also it can be applied to any dynamic system, with any time and spatial scale [2]. In such systems the causal loops are the main concept for model formulation. The causal loops represent the relation and interaction among the system variables. In the most cases in the SD approach these loops can't be described precisely and have vagueness and ambiguity. In such cases it is better to use SD with other methods. The fuzzy logic is a good suggestion for solving vague and imprecise problems in the SD approach; it is a technique with approximate reasoning which applies the linguistic descriptions to develop causal relationship between the input and output variables. Thus, overcomes the vague and imprecise problem, particularly where the mathematical model is not explicitly known.

In this paper the fuzzy logic is applied to define the relationship among the variables based on the if-then rules. For suitable analysis of SD methodology, we have used fuzzy logic for presenting vague conditions between variables. For this purpose, we have used of internal interaction of a national innovation system for analyzing creation and using knowledge loops in base of SD approach and we have compared its results with apply fuzzy logic to SD methodology. The rest of the paper is organized as follow. Section 2 focuses on the literature review and concept of SD approach and its components. Also fuzzy logic and Fuzzy Inference System (FIS) are mentioned in this section. In the section 3 the brief description of Research and Development (R\&D) and National System of Innovation (NSI) with procedures of building the FIS are represented. Section 4 illustrates the results of the suggested methodology, and finally conclusion.

\section{Literature Review}

In relation to SD researches, many applications in different fields which have diversity can be seen. For example system thinking and modeling for a complex world [1], applying system dynamics with data mining in a biological model [2], Navigating towards sustainable development: A system dynamics approach [3], Knowledge based decision making on higher level strategic concerns: system dynamics approach [4] and etc. Also the applications and implementations of fuzzy logic in different sciences and industrial problems are much because of its popularity to deals with vague and imprecise information [5], [6]. However the number of studies is focused on application of fuzzy logic in the system dynamics is not large amount. Pervious researches for this approach, which are considerable, are Tahera.k \& Ibrahim.R.N [6], Seç kin polat \& Cafer Erhan [7], Vassilios Karavezyris \& Klaus Peter [8].

\subsection{System Dynamics Approach}

SD as developed by Forrester [9] is a problem solving approach to complex problems which emphasize the structural aspects of model of systems. It can be used to modeling and simulation real world systems. SD deals with the time dependent behavior of managed systems with the aim of describing the system and understanding, through qualitative and quantitative models, how information feedback governs its behavior, and designing robust information feedback structures and control policies simulation and optimization [10]. SD consists of some components: Causal loop diagram, Stock and Flow diagram and Level and Rate variables.

\subsubsection{Causal Loop Diagrams}

The structure of a system in SD methodology is described by Causal Loop Diagrams (CLD). CLD represents the relationships and feedback loops among the variables in the system. In the CLD, influence of one variable on another variables represented by the arrows, which arrows with 
positive sign (+), show straight effect, as causal variable change and negative sign $(-)$, show an effected change is in the opposite direction of causal variable change. There are two common feedback mechanisms in CLD. These mechanisms are either negative feedback (balancing), or positive feedback (reinforcing) loops. The balancing loop illustrates goal seeking behavior. After a disturbance, the system seeks to return to an equilibrium situation. In a reinforcing loop initial disturbance allows system to have further change, and reaches an unstable equilibrium situation. Figure 1 depicts the CLD of new product introduction.

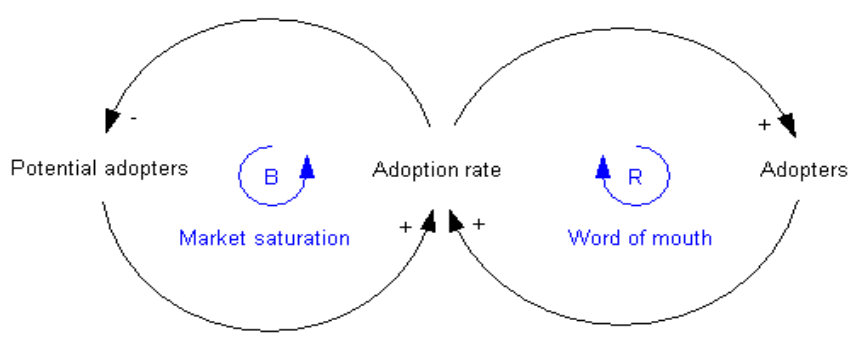

Figure 1. Causal loop diagram of new product introduction

The positive reinforcement (labeled $\mathrm{R}$ ) loop on the right indicates that increases in Adoption rate increases the number of Adopters, which again increases the overall Adoption rate. The second loop on the left, is balancing loop (labeled B), indicates that the greater the Adoption rate, causes decrease in Potential adopters, which again decreases Adoption rate. Consequently increases in Adoption rate, results in decreases of itself.

\subsubsection{Level and Rate variables}

The Level or Stock variables are the accumulations, i.e. the inventories within the system. They characterize the state of the system and generate the information upon which decisions and actions are based. Level variables give systems inertia and provide them with memory [1]. The rate variables represent the flow into or out of a level, which result from a decision making process.

\subsubsection{Stock and Flow diagrams}

CLD suffer from a number of limitations. One of the most important limitations of CLD is their inability to capture the stock and flow structure of systems. The model structure and interrelationship among the variables are represented by stock and flow diagram. Figure 2 exhibits the stock and flow diagram of new product adoption model. 


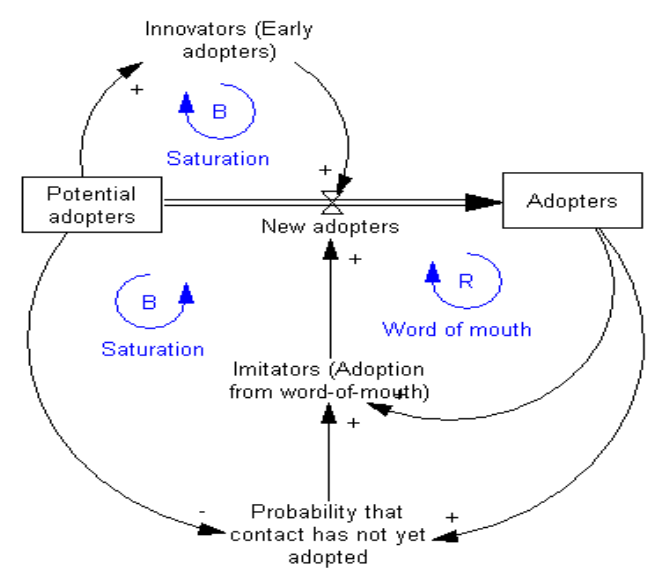

Figure 2. Stock and flow diagram of new product adoption

After structure the CLD and stock and flow diagrams, the next step is defining the mathematical equations among the variables. The below equations represent the relationship between stock and rate variables.

$$
\operatorname{stock}(t)=\int_{t_{0}}^{t}[\operatorname{Inflow}(s)-\operatorname{Outflow}(s)] d s+\operatorname{stock}\left(t_{0}\right)
$$

Where inflow ( $s$ ) represent the value of the inflow at any time ( $s$ ) between the initial time $\left(t_{0}\right)$ and the current time $(t)$. Equivalently, the net rate of change of any stock, its derivative, is the inflow minus the outflow, defining the differential equation

$$
d(\operatorname{stock}) / d t=\operatorname{Inflow}(t)-\operatorname{Outflon}(t)
$$

\subsection{Fuzzy Logic Approach}

Fuzzy sets were introduced by Zadeh [11] as an extension of the classical set theory. The fuzzy logic was built around the central concept of a fuzzy sets or membership functions. Fuzzy logic expresses vague and subjective relationship mathematically. It is a suitable way to mapping an input space to an output space using a fuzz set. The conventional set theory classifies the elements of the set into crisp set. The membership degree is One, when an element belongs to the set and zero, when an element does not belong to the set. Unlike the classical set theory, in the fuzzy set theory, an element can belong to a fuzzy set with the membership degree between zero and one. Figure 3 shows the difference between a classical set and a fuzzy set. In the classical set, 30C is considered as cold whereas in the fuzzy set, the degree of coldness for $30 \mathrm{C}$ is 0.85 and the degree of hotness for $30 \mathrm{C}$ is 0.15 , i.e. $30 \mathrm{C}$ is cold by $85 \%$ and hot by $15 \%$. 


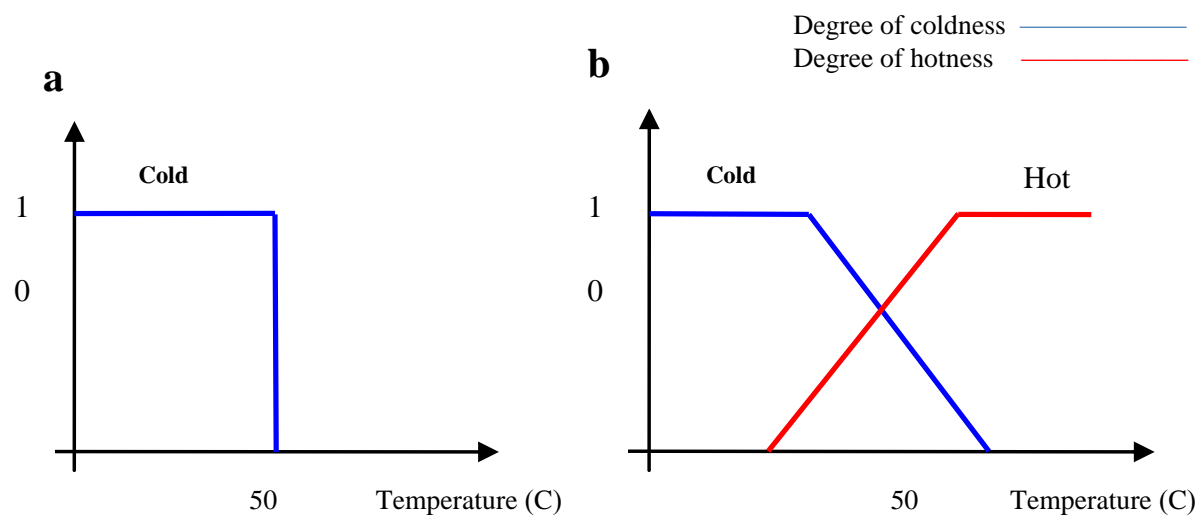

Figure 3. (a) Classical set and (b) Fuzzy set

A membership function is a curve that represents the degree of points which belong to the specific fuzzy variable. Selecting the appropriate membership function plays an essential rule in design of a fuzzy logic controller. The shape of membership function could be defined based on the simplicity, convenience, speed and efficiency. Many different membership functions are introduced in the literatures such as triangular, trapezoidal and Gaussian. The membership function which represented in figure $3(\mathrm{~b})$ is a trapezoidal type.

\subsubsection{Fuzzy Rule Based}

Fuzzy rule based is developed to relate the input variables to the output variables by if-then rules. Fuzzy rules consist of two parts: an antecedent part stating condition on the input variables, and a consequent part describing the related value of the output variables. A single fuzzy if-then rule assumes as follow:

If $x$ is $\mathrm{A}$ then $y$ is $\mathrm{B}$

Where $A$ and $B$, are linguistic values defined by fuzzy set on the ranges (universes of discourse) $X$ and $Y$, respectively. The if- part of the rule " $x$ is $\mathrm{A}$ " is antecedent or premise, while the thenpart of the rule " $y$ is $\mathrm{B}$ " is consequent or conclusion. An example such a rule might be

If service is good then price of food is average

In this rule, service and price are two fuzzy variables. The fuzzy values in this rule are good and average descriptions. These values are defined on universes of discourse and determine the degree of element $x$ which belongs to the membership functions. In general, the input to an if-then rule is the current value for the input variable (in this case, service) and the output is an entire fuzzy set (in this case, average). This set will later be defuzzified i.e. assigning one value to the output. The concept of defuzzification is described in the next section. In some case the antecedent or consequent of a rule can also have multiple parts. Therefore given particular values of the input variables, the degree of fulfillment of a rule is obtained by aggregating the membership degrees of these input values into the respective fuzzy sets. The fuzzy output is determined by the degrees of fulfillment and the consequent parts of the rules. The most common ways to modify the output fuzzy set are truncation using the min (minimum) function or scaling using the prod (product) function.

\subsubsection{Fuzzy Inference Systems}


Fuzzy inference is the process of formulating the mapping from given inputs to an output using fuzzy logic, the mapping then provides a basis from which a decision can be made. It used for evaluating fuzzy linguistic descriptions using concepts such as membership functions, fuzzy logic operators, and if-then rules [12]. Because of its multidisciplinary nature, FIS are associated with a number of names, such as fuzzy expert systems, fuzzy modeling, fuzzy associative memory, fuzzy logic controllers, and simply fuzzy systems. In general there are two common types of FIS: mamdani and sugeno types [5]. These two types of inference systems vary somewhat in the way outputs are determined. For these two types of FIS, there are entire descriptions in the literatures. [13], [14], [15].

The fuzzy inference diagram shown in figure 4 (MATLAB help, 2008) is the composite of all the smaller diagrams. It simultaneously displays all parts of the fuzzy inference process. Information flowing through the fuzzy inference diagram is shown in figure 4. The flow proceed up from the inputs in the lower left, then across each row, or rule, and then down the rule outputs to finish in the lower right.

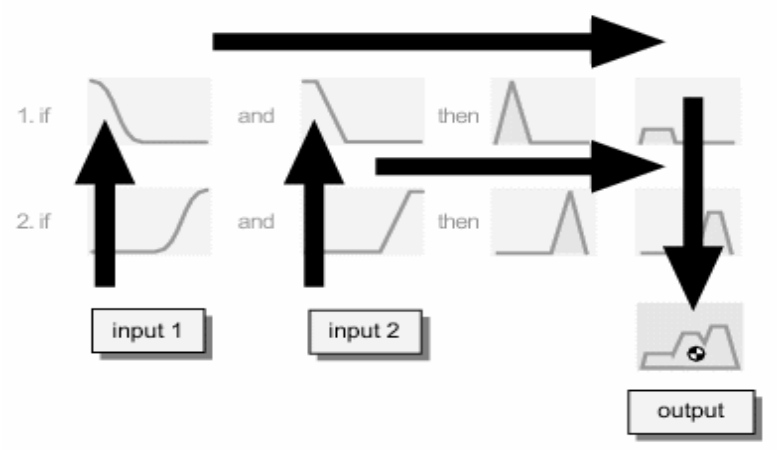

Figure 4. Fuzzy inference diagram

This figure employs the truncation method to modify the output of fuzzy set. This method is shown in the right column (result column). The min function which resulted from AND logic operator, is a common implication method in the mamdani type of FIS.

\section{Application of fuzzy logic for modeling System Dynamics}

If SD has at least one of the below conditions, can be modeled with fuzzy logic.

- Some of the level or rate or other variables are fuzzy.

- The time factor has vagueness.

- Some of the relations among variables can be replaced by conditional statements, which contain fuzzy variables. These conditional statements usually are in the if-then format.

- The degree of uncertainty of variables can be represented by fuzzy probabilities, when the information on hand, is imprecise or incomplete.

- Some of the operators may be fuzzy.

According to these properties the fuzzy logic approach is used to modeling SD. The SD model which applied in this study is the simulation of R\&D in the NSI. Figure 5 represents the stock and flow diagram of applying R\&D in the NSI. This diagram includes four level variables: R\&D output stock, Stock of absorbed knowledge, acquired knowledge stock and external knowledge stock, whereas the first two level variables mentioned are vital in our study. Also there are six rate variables that influences on the level variables. The rest of these variables are exogenous and auxiliary. This diagram follows directly from the causal loop diagram [16]. The purpose of this 
model is to simulate R\&D output generated in the system of innovation and to model and explain the effect the presence/lack of long term investment in R\&D and $R \& D$ resource could have on the system's ability to produce R\&D output [16]. prior to applying fuzzy logic to formulation of R\&D in the NSI, the brief description of concept of NSI and R\&D in national level presented in the following below.

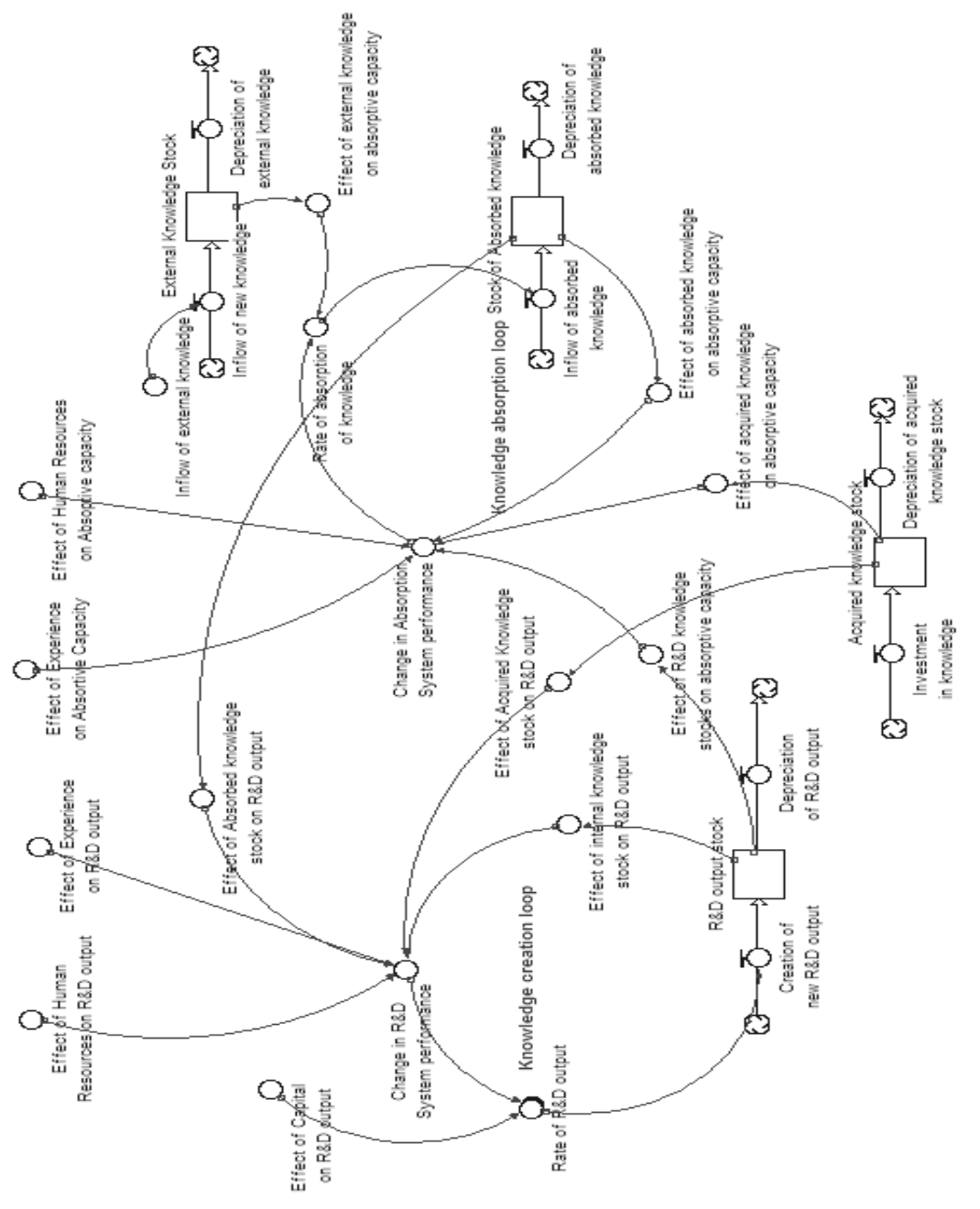

Figure 5. Stock and Flow Diagram with absorption and creation of knowledge

\subsection{Concept of R\&D and NSI}

Chris Freeman introduced the concept of a National System of Innovation to describe and interpret the performance of Japan [17]. He defined the NSI as the network of institutions of private and public sectors, whose activities and interactions initiate, import, modify, and diffuse new technologies. The NSI approach aims to identify key elements playing crucial rule in performing the systems functions. Freeman acknowledges the importance of R\&D in formal scientific and technical organizations within the NSI [17].

$R \& D$ is also introduced as a form of organizational learning. It plays a dual role within an economy. R\&D not only generates new information, but also enhances the ability to assimilate and exploit existing information [18]. 
A major advantage of $R \& D$ is its contribution to the current knowledge base. Cohen and Levinthal believe that since the long term investment in developing an R\&D capacity is substantial, it is not a trivial issue and is a matter of urgency that should be looked into [18].

\subsection{Building the FIS to modeling R\&D in the NSI}

In this section the steps of proposed approach are represented and the mamdani type inference process is adopted.

Step 1: Identification of variables

The first step in designing the fuzzy inference system is to identify fuzzy input and output variables of the controller. A common problem in the most studies is the selection of a limited number of variables that are relevant for the application, and for which reliable data can be obtained [19]. The input variable selection can be performed by means of data-driven techniques such as Artificial Neural Network, Principal Component or genetic algorithms [20], [21], [22], but has not yet been applied for rule based fuzzy model because these techniques often require a large dataset, and then the expert knowledge has mostly been used [23].

In this study the number of variables obtained based on analyzing the stock and flow diagram of $R \& D$ accurately. Consequently with take into accounting delay variable; there are sixteen input and ten output variables. Some of these variables are: R\&D output stock, creation of new R\&D, rate of R\&D output, stock of absorbed knowledge, inflow of absorbed knowledge, and rate of absorption knowledge and so on. These variables belong to the two main loops: Knowledge creation loop and Knowledge absorption loop, in the figure 5.

Step 2: Selecting fuzzy set and membership functions

After identification of fuzzy input and output variables, the next step is defining fuzzy set for each variable and selects the appropriate membership functions for the fuzzy variables. In this paper the trapezoidal membership function is employed to illustrate linguistic variables such as: very low, low, average, high and very high. We adopt for some of these fuzzy variables five and for the further variables, tree membership functions. Figure 6 depicts some of these membership functions with linguistic variables.
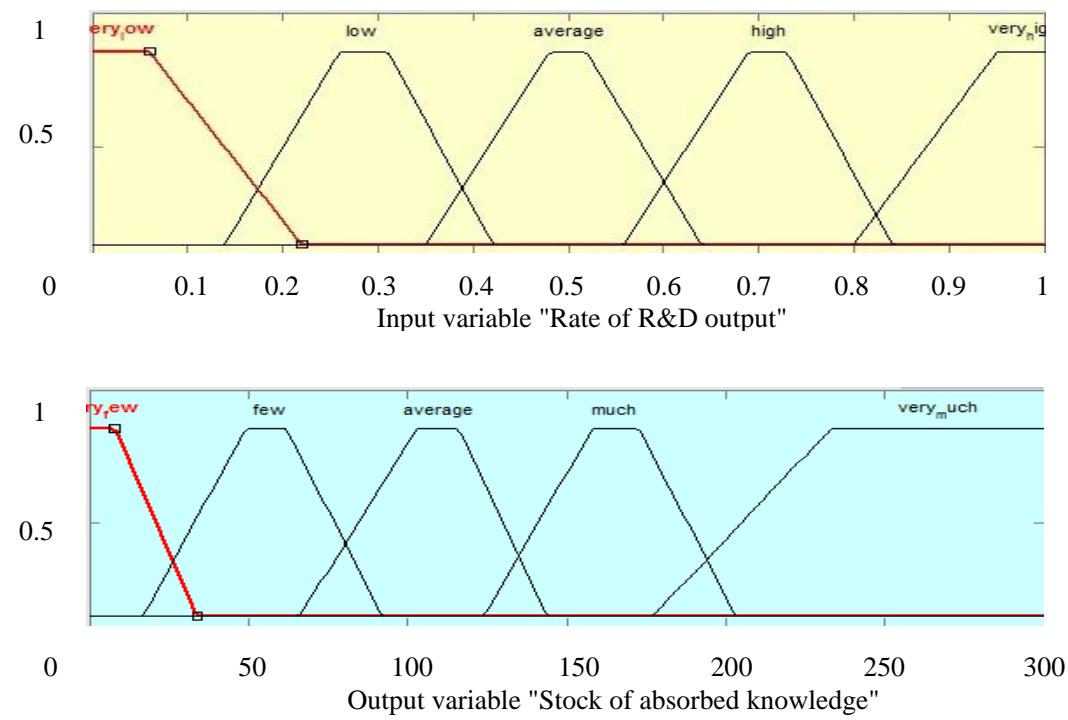

Figure 6. Membership functions of input and output variables. 
Step 3: Establish fuzzy rule base and set the inference process

Once the membership functions for each variable are structured, the fuzzy rule base is developed to relate the input variables to the output variables by if-then rules. The fuzzy operators such as " and" or "or" are applied across the rules that are built based on knowledge and experience about the Stock and Flow diagram in the figure 5. In this study fuzzy rule bases to modeling R\&D in the NSI are written in the seventy four rules to associate fuzzy input variables with the fuzzy output variables. Figure 7 illustrates some of these rules which are written by MATLAB Fuzzy Logic Toolbox.

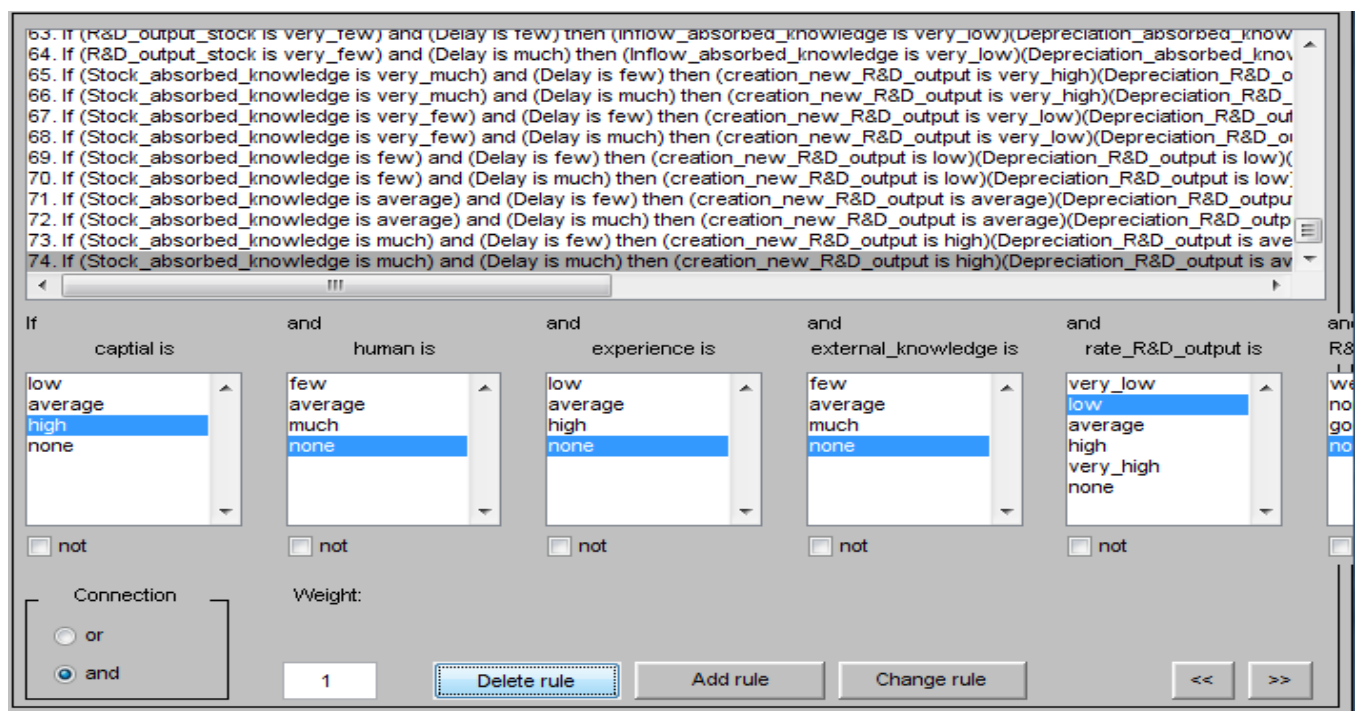

Figure 7. An example of the rules used in the FIS

We use mamdani type of FIS. Mamdani proposed a fuzzy implication rule for fuzzy control in 1977 [24]. The mamdani fuzzy logic operator is given as:

$$
f\left(\mu_{A}(x), \mu_{B}(y)\right)=\mu_{A}(x) \wedge \mu_{B}(x)
$$

Where $\mu_{A}(x)$ denotes the membership function of $x$.

In the mamdani implication, the "or" connective in a rule is replaced with the "max" operator and the "and" connective in a rule is replaced with the "min" operator. In addition the mamdani implication operator (i.e. min operator) is applied between the outcome of antecedent membership function and the consequent membership function. At the end, all the outputs are aggregated using aggregation methods, for instance maximum (max), probabilistic OR (probor), sum of the outputs (sum), and customized methods.

Step 4: Selecting the defuzzification methods

The final step is defuzzifying the fuzzy output variables into numeric values. The input for the defuzzification process is a fuzzy set and the output is a crisp single number. There are different defuzzification methods such as centroid, bisector, middle of the max, etc. The centroid method is applied in this study which returns the center of area under the curve.

After building the FIS to modeling the implementation of $R \& D$, the results of the FIS are considered. The following section represents the results of simulation.

\section{Model simulation result}


The fuzzy logic toolbox of MATLAB is used to design the FIS which computes the value of the output based on different input values. Figure 8 shows the curves which represent the mapping input variables to output variables. This figure illustrates there is a positive correlation between the input variable creation of new R\&D and the output variable R\&D output stock, and increases in input variable, increases the number of output variable.

Also in the $R \& D$ modeling by means of $S D$ approach, the creation of new $R \& D$ is a rate variable and represents flow into the level variable R\&D output stock; consequently enhancement in the entrance rate variable increases the number of level variable. This behavior in the figure 8(a) confirms the compatibility test in the SD approach which is one of the most model validation tests. Another relation depicted in the figure 8(b) is the influence of amount of R\&D output stock on its changing behavior. As the figure 8(b) represents if the R\&D output stock as an input variable increases, the number of R\&D output stock as an output variable will be increased, i.e. increases in the R\&D output stock results in later increases of itself.

In the SD method changes of the level variable based on the rate variable is a net growth or a net reduction, on the contrary in the proposed approach, this changes will not be a net growth or reduction and have an oscillation (figure 8). Therefore the proposed approach represents the behavior of the variable based on other variables in the more details during of simulation.

This is considerable if the number of associated if-then rules in the figure 7 increases, the curve which represented in the figure 8(a) has a more oscillation in the interval between 50 and 150, also in the figure 8(b) this changes will be happen in the interval between 200 and 300.

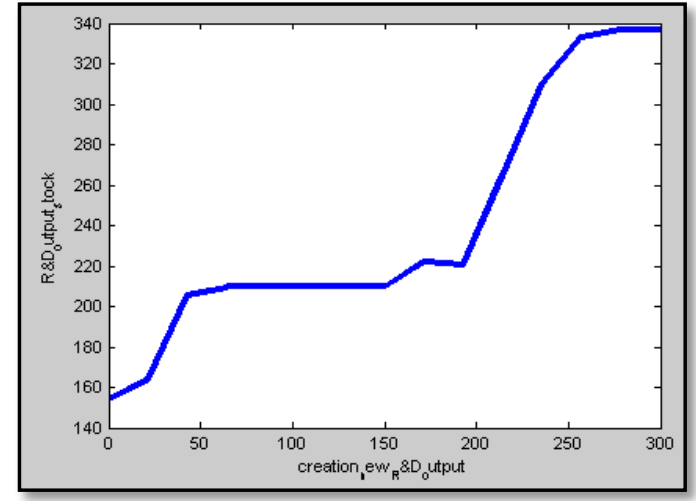

(a)

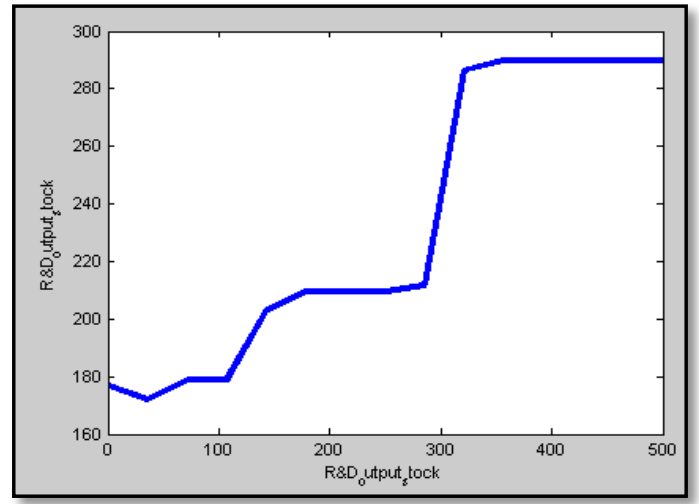

(b)

Figure 8. The relation between input and output variables (a) Effect of changing creation new R\&D on R\&D output stock and (b) Influence of amount of R\&D output stock on its changing behavior.

\section{Conclusion}

In the SD approach the effect of one variable to the others can be positive or negative and don't represent the qualitative relations and intensity of connections among the variables, while in the proposed approach this relations represented by means of linguistic variables such as "very high", "low", "very low" and so on which expresses the quality and intensity of relations. Specially, using of FISs helped us to analyze outcomes from SD methodology in a crisp manner with applying fuzzy variables within.

In this paper, we prepared a framework of NIS elements and only the two main loops are considered, the knowledge creation and knowledge absorption loop. In the future researches much and different loops can be considered. In such case we need to increase the number of input and output variables and the associated rules which define the behavior of systems completely. Also for 
Hassan Youssefi, Vahid Saeid Nahaei, Javad Nematian/ TJMCS Vol .2 No.1 (2011) 89-100

overcome problems e.g. input and output variables selection and optimization of number of rules and type of membership functions within the fuzzy rule based modeling, future researches could focus on hybridizing genetic algorithm and fuzzy logic as well as determining the optimal parameters of a complex systems. These future researches can verify the efficiency test in SD approach which is the one of common validation tests which analysis the complexity and optimality of model dimensions.

\section{Acknowledgement}

The authors wish to thank Ms. Roya Soltani for her help and support in making this study possible.

\section{Reference}

[1] Sterman, J.D., "Business Dynamics: System Thinking and Modeling for a Complex world", McGraw, 2000.

[2] Jeng, B., Chen, J.X., and Liang, T.P., "Applying data mining to learn system dynamics in a biological model", International Journal of Expert Systems with Applications, Vol. 30, No.1, pp. 50-58, 2006.

[3] Hjorth, P., and Bagheri, A., "Navigating towards sustainable development: A system dynamics approach", International Journal of Futures, Vol. 38, No. 1, pp. 74-92, 2006.

[4] Yim, N-H., Kim, S-H., Kim, H-W., and Kwahk, K-Y., "Knowledge based decision making on higher level strategic concern: system dynamic approach", International Journal of Expert Systems with Applications, Vol. 27, No. 1, pp. 143-158, 2004.

[5] Al-Najjar, B., and Alsyouf, I., "Selecting the most efficient maintenance approach using fuzzy multiple criteria decision making", International Journal of Production economics, Vol. 84, No. 1, pp. 85-100, 2003.

[6] Tahera, K., Ibrahim, R.N., and Lochert, P.B., "A fuzzy logic approach for dealing with qualitative quality characteristics of a process", International Journal of Expert Systems with Applications, Vol. 34, No. 4, pp. 26302638, 2008.

[7] Polat, S., and Bozdağ , C.E., "Comparison of fuzzy and crisp systems via system dynamics simulation", European Journal of Operational Research, Vol. 138, No. 1, pp. 178-190, 2002.

[8] Karavezyris, V., Timpe, K.P., and Marzi, R., "Application of system dynamics and fuzzy logic to forecasting of municipal solid waste", International Journal of Mathematics and Computers in Simulation, Vol. 60, No. 3-5, pp. 149-158, 2002.

[9] Forrester, J.W., "Industrial Dynamics", John Wiley \& Sons, 1961.

[10] Coyle, R.G., "System Dynamics Modeling", Chapman \& Hall: London, 1996.

[11] Zadeh, L.A., "Fuzzy sets", Information and control, Vol. 8, No. 3, pp. 338-353, 1965.

[12] Tsoukalas, L., and Uhrig, R., "Fuzzy and Neural Applications in Engineering", John Wiley, 1997.

[13] Jang, J.S.R., and., Sun, C.T., "Neuro-Fuzzy and Soft computing: A Computational Approach to Learning and Machine Intelligence", Prentice Hall, 1997.

[14] Mamdani, E.H., and Assilian, S., "An experiment in linguistic synthesis with a fuzzy logic controller", International Journal of Man-Machine Studies, Vol. 7, No. 1, pp. 1-13, 1975.

[15] Takagi, T., and Sugeno, M., "Fuzzy identification of systems and its applications to modeling and control", IEEE Transactions of System. Man Cybernetics, Vol. 15, No. 1, pp. 116-132, 1985.

[16] Grobbelaar, S.S., "R\&D in the National System of Innovation: A System Dynamics Model", PhD Dissertation, University of pretoria, 2006.

[17] Freeman, C., "Technology Policy and Economic Performance. Lessons from Japan", Pinter: London, 1987.

[18] Cohn, W.M., and Levinthal, D.A., "Absorptive Capacity: A new perspective on learning and innovation", Administrative Science Quarterly, Vol. 35, No., pp. 128-152, 1990.

[19] Maier, H.R., and Dandy, G.C., "Neural network for the prediction and forecasting of water resource variables: a review of modeling issues and applications", Environ Model Software, Vol. 15, pp. 101-124, 2000.

[20] Holland, J.H., "Adaptation in natural and artificial systems", MI: University of Michigan Press, 1975.

[21] Joliffe, I.T., "Principal component analysis", New York: Springer Verlag, 1986.

[22] Goldberg, D.E., "Genetic algorithm in search, optimization and machine learning", MA: Addison-Wesley Publishing Company, 1989. 
Hassan Youssefi, Vahid Saeid Nahaei, Javad Nematian/ TJMCS Vol .2 No.1 (2011) 89-100

[23] Salski, A., "Ecological modeling and data analysis", In: Zimmermann, H.J., "practical applications of fuzzy technologies", Kluwer-Nijhoff Publishing, pp. 247-266, 1999.

[24] Mamdani, E.H., "Application of fuzzy logic to approximate reasoning using linguistic synthesis", IEEE Trans. Comput, Vol. 26, pp. 1182-1191, 1977. 